TRANSACTIONS OF THE

AMERICAN MATHEMATICAL SOCIETY

Volume 280, Number 2, December 1983

\title{
ALL VARIETIES OF CENTRAL COMPLETELY SIMPLE SEMIGROUPS
}

BY

\author{
MARIO PETRICH AND NORMAN R. REILLY
}

\begin{abstract}
Completely simple semigroups may be considered as a variety of algebras with the binary operation of multiplication and the unary operation of inversion. A completely simple semigroup is central if the product of any two idempotents lies in the centre of the containing maximal subgroup. Central completely simple semigroups form a subvariety $\mathcal{C}$ of the variety of all completely simple semigroups. We find an isomorphic copy of $\mathcal{L}(e)$ as a subdirect product of the lattices $\mathcal{L}(\mathscr{Q} R \mathcal{G})$, $\mathcal{L}(\mathcal{Q} \mathcal{G})$, and $\mathcal{L}(\mathcal{G})$ of all varieties of rectangular bands, abelian groups, and groups, respectively. We consider also several homomorphisms and study congruences they induce.
\end{abstract}

1. Introduction and summary. The class of completely simple semigroups considered as algebras with the binary operation of multiplication and the unary operation of inversion forms a variety determined by a small set of simple axioms. The study of the lattice of subvarieties of this variety was made possible by the description of its free objects due to Clifford [1] and Rasin [9]. An investigation of the varieties of completely simple semigroups has recently been initiated by Rasin [9] and the authors [6,7]. In the last-mentioned reference, the variety $C$ of all central completely simple semigroups showed some remarkable properties. (A band of groups was called central by Leech [3] if the product of any two idempotents lies in the centre of the containing maximal subgroup.) Maševickiǐ [4] and Rasin [8] determined all subvarieties of the variety $\mathbb{Q}$ of completely simple semigroups with abelian subgroups; this result was proved by yet another method in [9].

All subvarieties of the variety $\Re \mathcal{G}$ of rectangular groups are easy to find. It was proved in [7] that $\mathscr{Q} \vee \Re \mathcal{G}=\mathcal{C}$. We determine here all subvarieties of $\mathcal{C}$ thereby extending the work of Maševickii [4] and Rasin [8]. Our description of the lattice $\mathcal{L}(\mathcal{C})$ of all subvarieties of $\mathcal{C}$ is similar to that of the cited authors for the lattice of all subvarieties of $Q$.

$\$ 2$ contains all the material from [6 and 7] which is needed in later sections. The main characterization theorem can be found in $\S 3$, viz., an embedding of $\varrho(\mathcal{C})$ into the direct product $\mathscr{L}(\mathscr{R}) \times \mathcal{L}(\mathscr{Q}) \times \mathcal{L}(\mathcal{G})$, where $\mathscr{R} \mathscr{B}$, and $\mathcal{Q S}$ and $\mathcal{G}$ stand for the varieties of rectangular bands, abelian groups and groups, respectively; we actually consider a homomorphism on a larger class of varieties of completely simple

Received by the editors October 7, 1982.

1980 Mathematics Subject Classification. Primary 20M05, 20M10.

Key words and phrases. Completely simple semigroups, varieties, central completely simple semigroups, fully invariant normal subgroups, free completely simple semigroups.

(C)1983 American Mathematical Society $0002-9947 / 83 \$ 1.00+\$ .25$ per page 
semigroups. Various additional information concerning this embedding makes up $\S 4$. Isomorphisms of various intervals of the lattice $\mathcal{L}(\mathcal{C})$ are considered in $\S 5$, which provide further insight into the structure of the lattice $\mathcal{L}(\mathcal{C})$.

2. Preliminaries. In general, we use the notation and terminology of Howie [2] or Petrich [5]. In particular, we adopt the notation in [5] for Rees matrix semigroups, and use the description of congruences on a Rees matrix semigroup presented in [2]. In order to minimize the typographical complexity we modify the standard notation for a sandwich matrix and denote the $(j, k)$ th entry by $[j, k]$.

We will consistently use the following notation:

$\mathcal{G}$ - the variety of all groups,

$Q \mathcal{G}$ - the variety of all abelian groups,

$\mathfrak{T}$ - the variety of trivial groups,

$\Re G_{3}$ - the variety of all rectangular bands,

$\mathscr{R} \mathcal{G}$ - the variety of all rectangular groups (orthodox completely simple),

ES - the variety of all completely simple semigroups,

$[Q, \mathscr{B}]$ - the interval of a lattice with minimum $\mathbb{Q}$ and maximum $\mathscr{B}$,

$\widetilde{T}_{X}$-the semigroup of all transformations on a set $X$,

$\mathcal{L}(\mathcal{V})$ - the lattice of all subvarieties of a variety $\mathcal{T}$ of completely simple semigroups,

End $S$ - the semigroup of all endomorphisms of a semigroup $S$,

$\langle\mathscr{K}\rangle$ - the variety of all completely simple semigroups generated by the class $\mathscr{K}$ of completely simple semigroups, and write $\langle S\rangle$ if $\mathscr{K}=\{S\}$,

[ $E_{S}$ ]-the semigroup generated by the set $E_{S}$ of all idempotents of a semigroup $S$ ([ $\left.E_{S}\right]$ ) is completely simple if $S$ is),

$\left[u_{\alpha}=v_{\alpha}\right]_{\alpha \in A}$ - the variety of completely simple semigroups defined by the laws $u_{\alpha}=v_{\alpha}, \alpha \in A$,

[ $H, K]$ - the subgroup generated by the elements of the form $h^{-1} k^{-1} h k$ where $h \in H, k \in K$ and $H$ and $K$ are subgroups of a group $G$,

$G^{\prime}=[G, G]$ - the commutator subgroup of $G$,

$\hat{H}$ - the normal closure of the subgroup $H$,

$\mathcal{Q}(G)$ - the smallest normal subgroup (which is necessarily fully invariant) of the group $G$ such that $G / \mathcal{U}(G)$ is a member of the group variety $\mathcal{Q}$,

$x^{0}=x x^{-1}$, for $x \in S, S$ a completely simple semigroup.

Lemma 2.1 [9]. Let $S=\mathfrak{N}(I, G, \Lambda ; P)$, where $P$ is normalized. Let $\varphi \in \widetilde{T}_{I}$, $\omega \in$ End $G, \psi \in \mathcal{T}_{\Lambda}$ be such that

$$
[\lambda, i] \omega=[1 \psi, 1 \varphi][\lambda \psi, 1 \varphi]^{-1}[\lambda \psi, i \varphi][1 \psi, i \varphi]^{-1} \quad(\lambda \in \Lambda, i \in I)
$$

Then $\theta=\theta(\varphi, \omega, \psi)$ defined by

$$
(i, g, \lambda) \theta=\left(i \varphi,[1 \psi, i \varphi]^{-1}(g \omega)[1 \psi, 1 \varphi][\lambda \psi, 1 \varphi]^{-1}, \lambda \psi\right),
$$

is an endomorphism of $S$. Conversely, every endomorphism of $S$ can be so written uniquely. 
A construction of the Rees matrix representation of a free completely simple semigroup follows.

Lemma $2.2[1,9]$. Let $X=\left\{x_{i} \mid i \in I\right\}$ be a nonempty set, fix $1 \in I$ and let $I^{\prime}=I \backslash\{1\}$. Let $Z=\left\{q_{i} \mid i \in I\right\} \cup\left\{[j, k] \mid j, k \in I^{\prime}\right\}, F_{Z}$ be the free group on $Z$, and let $P=([j, k])$ with $[1, k]=[j, 1]=1$, the identity of $F_{Z}$. Then $F=\mathfrak{N}\left(I, F_{Z}, I ; P\right)$ is a free completely simple semigroup over $X$, with embedding $x_{i} \rightarrow\left(i, q_{i}, i\right)$.

Notation 2.3. We fix a countably infinite set $X$, and in addition to the above notation, introduce

$$
F_{q}=\left\langle q_{i} \mid i \in I\right\rangle, \quad F_{p}=\left\langle[j, k] \mid j, k \in I^{\prime}\right\rangle,
$$

the free subgroups of $F_{7}$ generated by the sets $\left\{q_{i} \mid i \in I\right\}$ and $\left\{[j, k] \mid j, k \in I^{\prime}\right\}$, respectively. We will consistently use the notation $F=\Re\left(I, F_{Z}, I ; P\right)$ introduced above.

We will need only fully invariant idempotent separating congruences, for they are precisely the ones which correspond to the varieties in the interval [ $\Re, \mathcal{S}$ ]. In this context, the following special case of [2, Lemma 4.19] is of particular interest.

Lemma 2.4. Let $S=$ ơ $(I, G, \Lambda ; P)$. If $N$ is a normal subgroup of $G$, then $\rho_{N}$ defined on $S$ by

$$
(i, g, \lambda) \rho_{N}(j, h, \mu) \Leftrightarrow i=j, \quad g h^{-1} \in N, \quad \lambda=\mu,
$$

is an idempotent separating congruence on $S$, and every such congruence is obtained in this way. Writing $P / N$ for the $\Lambda \times I$ matrix with the $(j, k)$ th entry equal to the $(j, k)$ th entry of $P$ modulo $N, S / \rho$ is isomorphic to $\Re(I, G / N, \Lambda ; P / N)$.

Notation 2.5. We will consistently use the notation $\rho_{N}$ introduced above. For a variety $\mathcal{T}$ of completely simple semigroups, we denote by $\rho(\mathcal{T})$ the fully invariant congruence on $F$ corresponding to $\tau$. Also let

$$
\begin{aligned}
& \Re\left(F_{Z}\right)=\left\{\omega \in \text { End } F_{Z} \mid \text { there exist } \varphi, \psi \in \mathcal{T}_{I} \text { such that }(1) \text { holds }\right\}, \\
& \&\left(F_{p}\right)=\left\{\psi \in \text { End } F_{p} \mid \text { there exist } \varphi, \psi \in \mathcal{T}_{I} \text { such that }(1) \text { holds }\right\} .
\end{aligned}
$$

Hence $\varepsilon\left(F_{Z}\right)$ consists precisely of endormorphisms of $F_{Z}$ that arise in association with endomorphisms of $F$. The latter are uniquely determined by the functions $\left\{q_{i} \mid i \in I\right\} \rightarrow F_{Z}, \varphi, \psi \in \mathcal{T}_{I}$ independently. Furthermore, $\mathcal{E}\left(F_{p}\right)$ consists precisely of endomorphisms of $F_{p}$ that extend to elements of $\mathcal{E}\left(F_{Z}\right)$.

LEMMA 2.6 [9]. Let $N$ be a normal subgroup of $F_{Z}$. Then $\rho_{N}$ is fully invariant if and only if $N \omega \subseteq N$ for all $\omega \in \mathcal{E}\left(F_{Z}\right)$.

Definition 2.7. A normal subgroup of $F_{Z}$ (respectively, $F_{p}$ ) is $\varepsilon$-invariant if it is invariant under all $\omega \in \mathcal{E}\left(F_{Z}\right)$ (respectively, $\mathcal{E}\left(F_{p}\right)$ ). The set of all $\mathcal{E}$-invariant

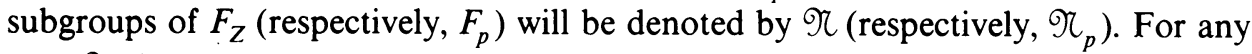
$N \in \Re$, let $N_{q}=N \cap F_{q}$ and $N_{P}=N \cap F_{p}$.

It is clear that $\Re$ (respectively, $\Re_{p}$ ) is a sublattice of the lattice of all normal subgroups of $F_{Z}$ (respectively, $F_{p}$ ), and that each element of $\mathscr{N}_{p}$ is the intersection with $F_{p}$ of an element of $\Re$ (e.g., its normal closure in $F_{Z}$ ). 
Proposition 2.8 [9]. The interval [ $\Re \Re, ~ อ S]$ is anti-isomorphic to the lattice 0 ). In particular, $\mathscr{V} \in[\mathscr{G} \mathscr{G}, \mathrm{S}]$ if and only if $\rho(\mathcal{T})$ is idempotent separating and so is of the form $\rho_{N}, N \in \mathfrak{X}$. The mapping $\rho(\mathfrak{V}) \rightarrow N$, where $\rho(\mathfrak{V})=\rho_{N}$, is an isomorphism of the lattice of fully invariant idempotent separating congruences on $F$ onto $\mathcal{X}$.

Lemma 2.9 [6]. If $N \in \mathcal{X}$, then $N_{q}$ is a fully invariant subgroup of $F_{q}$. If $\mathcal{T} \in \mathcal{L}(\mathfrak{S})$ is such that $\rho(\mathfrak{V})=\rho_{N}$, then $\mathfrak{V} \cap \mathcal{G}=\left\langle F_{q} / N_{q}\right\rangle$.

Notation 2.10. Let $\pi_{q}$ and $\pi_{p}$ be the projections of $F_{Z}$ onto $F_{q}$ and $F_{p}$, respectively.

LEMMA 2.11. (i) $\pi_{q}, \pi_{p} \in \mathcal{E}\left(F_{Z}\right)$.

(ii) If $N \in \mathcal{N}$, then $N \pi_{q}=N_{q}, N \pi_{p}=N_{p}$.

(iii) $\pi_{p}$ induces a homomorphism of the lattice $\Re$ onto the lattice $\Re_{p}$.

Proof. Parts (i) and (ii) were established in [6]. It was also shown in [6, Lemma 4.2(iii)] that $\pi_{q}$ induces a homomorphism of $\mathfrak{N}$ onto the lattice of fully invariant subgroups of $F_{q}$. A similar argument applied to $\pi_{p}$ will establish (iii).

THEOREM 2.12 [6]. The mapping $\chi: \mathfrak{T} \rightarrow \mathfrak{V} \cap \mathcal{G}$ is a homomorphism of $\mathcal{L}(\mathcal{C S})$ onto $\mathcal{L}(\mathcal{Q})$. The congruence $\alpha$ induced by $\chi$ may be characterized on $[\Re \mathscr{R}, \varrho S]$ as follows: for $\mathscr{Q l}, \mathscr{V} \in[\mathfrak{R} \mathscr{G}, \mathcal{C S}]$ with $\rho(\mathcal{Q})=\rho_{M}, \rho(\mathscr{V})=\rho_{N}$,

$$
\text { Q } \alpha \mathscr{V} \Leftrightarrow M_{q}=N_{q} \text {. }
$$

In particular, $(\mathcal{Q}, \mathcal{G}) \in \alpha$ if and only if $M_{q}=\{1\}$.

Three varieties considered in [7] will figure prominently here.

DEFINITION 2.13. A completely simple semigroup $S$ is central if the product of any two idempotents of $S$ lies in the centre of the containing maximal subgroup. The class of all central completely simple semigroups will be denoted by $C$.

Proposition 2.14 [7]. (i) $e=\left[a x^{0} a^{0} y a=a y a^{0} x^{0} a\right]$.

(ii) $e$ consists of all those completely simple semigroups for which the sandwich matrix entries in some (and consequently, every) normalized Rees matrix representation lie in the centre of the structure group.

(iii) $\rho(\mathcal{C})=\rho_{K}$, where $K=\left[F_{Z}, F_{p}\right]$.

Notation 2.15 . Let $Q$ denote the class of all completely simple semigroups with abelian subgroups.

Proposition 2.16 [17]. (i) $Q=[$ axaya = ayaxa $]$.

(ii) $\rho(\mathbb{Q})=\rho_{F_{Z}^{\prime}}$.

Notation 2.17. We write $\mathscr{D}=\left[a x^{0} a^{0} y^{0} a=a y^{0} a^{0} x^{0} a\right]$.

PROPOSITION 2.18 [7]. (i) D consists of all those completely simple semigroups for which the sandwich matrix entries in some (and consequently, every) normalized Rees matrix representation commute.

(ii) $\rho(\mathscr{Q})=\rho_{L}$ where $L=\hat{F}_{p}^{\prime}$.

It is clear that $Q \subseteq \mathcal{C} \subseteq \mathcal{D}$.

We conclude this section with a well-known universal algebraic result adapted to the present context. 
LEMMA 2.19. For any class $\mathscr{K}$ of completely simple semigroups

$\langle\mathcal{K}\rangle=\left\{S \in \mathcal{C} \mathcal{S} \mid \exists S_{\alpha} \in \mathcal{K}, T \subseteq \Pi S_{\alpha}\right.$ and an epimorphism $\left.\theta: T \rightarrow S\right\}$. In particular, for $\mathcal{Q}, \mathcal{T} \in \mathcal{L}(\mathrm{eS})$,

$$
\begin{aligned}
\mathscr{Q} \vee \mathcal{V} & =\langle\mathscr{Q} \cup \mathcal{T}\rangle \\
& =\{S \in \mathcal{C} \mid \exists U \in \mathcal{Q}, V \in \mathscr{V} \text { and an epimorphism } \theta: T \rightarrow S \\
& \text { for some subdirect product } T \text { of } U \times V\} .
\end{aligned}
$$

3. An embedding of $\mathcal{L}(\mathcal{C})$. We aim here at the principal result of the paper, viz., an embedding of $\mathscr{L}(\mathcal{C})$ into $\mathscr{L}(\Re \mathscr{B}) \times \mathscr{L}(\mathscr{Q}) \times \mathscr{L}(\mathcal{G})$. The proof is broken into several auxiliary results, some of which are valid in greater generality than that needed for the present purpose, and some of which are of independent interest.

LEMMA 3.1. The mapping

$$
\delta: \mathcal{T} \rightarrow \mathscr{T} \cap \mathscr{R} \mathscr{R} \quad(\mathcal{V} \in \mathcal{L}(e S))
$$

is a homomorphism of $\mathcal{L}(\mathcal{S})$ onto $\mathcal{L}(\Re \Re)$.

Proof. Let $\mathscr{Q}, \mathcal{V} \in \mathcal{L}(\mathcal{C S})$ and $S \in(\mathcal{Q} \vee \mathcal{V}) \cap \Re \Re$. By Lemma 2.19, there exists a subdirect product $T \subseteq U \times V$, where $U \in \mathcal{U}$ and $V \in \mathcal{T}$, and an epimorphism $\varphi: T \rightarrow S$. Let $T^{\prime}=\left\{\left(H_{u}, H_{v}\right) \mid(u, v) \in T\right\}$ and let $\left(H_{u}, H_{v}\right)=\left(H_{a}, H_{b}\right) \in$ $T^{\prime}$. Then $u u^{-1}=a a^{-1}$ and $v v^{-1}=b b^{-1}$ which implies

$$
\begin{aligned}
(u, v) \varphi & =(u, v) \varphi[(u, v) \varphi]^{-1}=\left(u u^{-1}, v v^{-1}\right) \varphi \\
& =\left(a a^{-1}, b b^{-1}\right) \varphi=(a, b) \varphi[(a, b) \varphi]^{-1}=(a, b) \varphi,
\end{aligned}
$$

since $S$ is a rectangular band. It then follows that

$$
\varphi^{\prime}:\left(H_{u}, H_{v}\right) \rightarrow(u, v) \varphi \quad\left(\left(H_{u}, H_{v}\right) \in T^{\prime}\right)
$$

is single-valued and thus a homomorphism of $T^{\prime}$ onto $S$. Since $U / \mathcal{H} \in \mathcal{Q} \cap \Re \mathscr{B}$ and $V / \mathcal{H} \in \mathcal{T} \cap \Re \mathscr{R}$, and $T^{\prime}$ is a subdirect product of $U / \mathcal{H}$ and $V / \mathcal{H}$; it follows that $S \in(\mathscr{R} \cap \Re \Re) \vee(T \cap R \Re)$. Consequently,

$$
(\mathscr{T} \vee \mathfrak{T}) \cap \Re \Re \subseteq(\mathscr{R} \cap \Re) \vee(\mathscr{V} \cap \Re \Re)
$$

and the opposite inclusion is trivial. Therefore $\delta$ is a (lattice) homomorphism.

Our next objective is to obtain a homomorphism of $\mathcal{L}(\mathcal{C})$ into the lattice $\mathcal{L}(\mathscr{Q} \mathcal{G})$ of varieties of abelian groups.

Lemma 3.2. If $N \in \mathcal{N}$ and $F_{p}^{\prime} \subseteq N_{p}$, then $N_{p}$ is a fully invariant subgroup of $F_{p}$.

Proof. Since $F_{p}^{\prime} \subseteq N_{p}$, any element $n \in N_{p}(n \neq 1)$ can be written in the form

$$
n=\left[i_{1}, j_{1}\right]^{r_{1}} \ldots\left[i_{k}, j_{k}\right]^{r_{k}} g
$$

where $g \in F_{p}^{\prime} \subseteq N_{p}$ and the elements $\left[i_{t}, j_{t}\right]$ are distinct and $r_{t} \neq 0(t=1, \ldots, k)$.

Let $r$ denote the smallest positive exponent $r_{i}$ appearing in an expression of the form (2) for some $n \in N_{p}$. Without loss of generality $r=r_{1}$ in (2).

Let $k, l \in I$ and define $\varphi, \psi \in \sigma_{I}$ by

$$
i \varphi=\left\{\begin{array}{ll}
k & \text { if } i=i_{1}, \\
1 & \text { otherwise, }
\end{array} \quad j \psi= \begin{cases}l & \text { if } j=j_{1}, \\
1 & \text { otherwise }\end{cases}\right.
$$


Now let $\lambda \in \varepsilon\left(F_{7}\right)$ be defined by (1) and (3) on the free generators of $F_{p}$ and to act identically on $\left\{q_{i} \mid i \in I\right\}$. Then

$$
[i, j] \lambda= \begin{cases}{[k, l]} & \text { if }[i, j]=\left[i_{1}, j_{1}\right] \\ 1 & \text { otherwise }\end{cases}
$$

Since $n \lambda \in N_{p}$ and $g \lambda \in F_{p}^{\prime} \subseteq N_{p}$ ( $F_{p}^{\prime}$ is fully invariant in $F_{p}$ ), it follows from (2) that $[k, l]^{r} \in N_{p}$, for all $k, l \in I$. By the minimality of $r$ it now follows that $r$ divides each $r_{i}$ in the expression (2) and therefore $N_{p}$ must consist precisely of all elements of the form

$$
\left[i_{1}, j_{1}\right]^{s_{1} r} \cdots\left[i_{k}, j_{k}\right]^{s_{k} r} g
$$

where the $s_{t}$ are integers and $g \in F_{p}^{\prime}$. This is easily seen to be a fully invariant subgroup of $F_{p}$.

Lemma 3.3. Let $\left[F_{Z}, F_{p}\right] \subseteq N \in \mathcal{~} \mathfrak{X}$. Then $N=N_{q}\left[F_{Z}, F_{p}\right] \hat{N} N_{p}$.

Proof. By hypothesis, it is clear that $N_{q}\left[F_{Z}, F_{p}\right] \hat{N} N_{p} \subseteq N$. On the other hand, for any $n \in N, n=a_{1} b_{1} \cdots a_{k} b_{k}$ for some $a_{i} \in F_{q}, b_{i} \in F_{p}$. By applying $\pi_{q}$ and $\pi_{p}$ we find that $a_{1} \cdots a_{k} \in N_{q}$ while $b_{1} \cdots b_{k} \in N_{p}$. Hence

$$
\begin{aligned}
n & =a_{1} b_{1} \cdots a_{k} b_{k} \\
& =a_{1} b_{1} a_{2} b_{1}^{-1} a_{2}^{-1} a_{2} b_{1} b_{2} a_{3} \cdots a_{k} b_{k} \\
& =a_{1}\left(b_{1}\left[a_{2}^{-1}, b_{1}\right] b_{1}^{-1}\right) a_{2} b_{1} b_{2} a_{3} \cdots a_{k} b_{k} \\
& =a_{1} g_{1} a_{2} b_{1} b_{2} a_{3} \cdots a_{k} b_{k} \\
& =\cdots \\
& =a_{1} g_{1} a_{2} g_{2} a_{3} \cdots g_{k} b_{1} \cdots b_{k}
\end{aligned}
$$

where $g_{i} \in\left[F_{Z}, F_{p}\right]^{\hat{n}}$, and so, continuing

$$
n=a_{1} a_{2} \cdots a_{k} h_{1} \cdots h_{k} b_{1} \cdots b_{k}
$$

where $h_{i} \in\left[F_{Z}, F_{p}\right]^{\hat{1}}$. Thus $n \in N_{q}\left[F_{Z}, F_{p}\right] \hat{N_{p}}$, as required.

The homomorphism that we are seeking will arise from consideration of the idempotent generated elements of a variety. For this we introduce

Notation 3.4. For $\mathcal{T} \in \mathcal{E}(\mathcal{S})$, we will write $\mathscr{G} \mathcal{V}$ for the class of idempotent generated members of $\mathcal{T}$.

The classes $\mathscr{T} \mathcal{V}$, for $\mathcal{V} \in \mathcal{L}(\mathcal{E S})$, were considered in some detail by the authors in [7], where they were termed near varieties.

Definition 3.5. Let $\mathcal{C}$ be a class of completely simple semigroups. A semigroup $S$

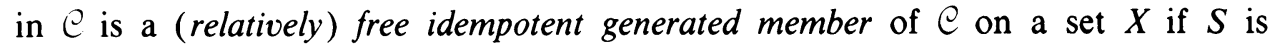
generated by a set $\left\{e_{x} \mid x \in X\right\}$ of idempotents and, for any mapping $\varphi$ of $X$ onto a set of idempotent generators of a semigroup $T$ in $\mathcal{C}$, there exists a unique homomorphism $\theta$ of $S$ onto $T$ such that $e_{x} \theta=x \varphi$ for all $x \in X$.

A free idempotent generated member of $\mathscr{T} \mathscr{V}(\mathscr{V} \in[\Re \Re, C \delta])$ was described as follows in [7, Theorem 3.2]. 
Lemma 3.6. Let $\mathscr{V} \in[\Re \Re, \mathcal{C S}]$ and $\rho(\mathcal{V})=\rho_{N}$. Then $S=\Re\left(I, F_{p} / N_{p}, I ; P / N_{p}\right)$ is a free idempotent generated member of $\mathscr{V}$ (and, therefore, also of $\mathscr{G} \mathcal{V}$ ) on the set $X=\left\{x_{i} \mid i \in I\right\}$. A set of idempotents generating $S$ for this purpose is

$$
\left\{\left(i,[i, i]^{-1}, i\right) \rho_{N} \mid i \in I\right\} \text {. }
$$

Proposition 3.7. For $\mathscr{V} \in \mathcal{L}(\mathcal{C S})$ with $\rho(\mathcal{V})=\rho_{N},\langle\mathcal{G}\rangle \cap G=\left\langle F_{p} / N_{p}\right\rangle$. If $\mathscr{T} \in \mathcal{L}(\mathscr{D})$, then in addition $\langle\mathscr{G} \mathcal{V}\rangle \cap \mathcal{G}=\{G \in \mathcal{G} \mid G$ is a subgroup of some $S \in \mathscr{G} \mathcal{V}\}$.

Proof. That $F_{p} / N_{p} \in\langle\mathscr{G}\rangle \cap \mathcal{G}$ is clear from Lemma 3.6 and so $\left\langle F_{p} / N_{p}\right\rangle \subseteq$ $\langle\mathscr{G}\rangle \cap \mathcal{G}$. Let $G \in\langle\mathscr{G}\rangle \cap \mathcal{G}$. Then, for some $S_{k} \in \mathscr{G} \mathcal{V}$ and a completely simple subsemigroup $R$ of $S=\Pi\left\{S_{k} \mid k \in K\right\}$, there exists an epimorphism $\varphi$ of $R$ onto $G$. Let $H$ be any countably generated subgroup of $G$. Then $H$ is the image under $\varphi$ of a countably generated completely simple subsemigroup $R^{*}$ of $R$. Since each $S_{k}$ is generated by idempotents, $R^{*}$ is a subsemigroup of a completely simple subsemigroup $R^{* *}$ of $T$ generated by a countable number of idempotents. Now $R^{* *} \in \mathscr{T}$ V and so, by Lemma 3.6, is a homomorphic image of $\Re\left(I, F_{p} / N_{p}, I ; P / N_{p}\right)$, a free object in $\mathscr{T}$ on a countable number of generators. Hence the subgroups of $R^{* *}$ must lie in $\left\langle F_{p} / N_{p}\right\rangle$, as must the subgroups of $R^{*}$ and consequently $H$ also. By the arbitrariness of $H, G \in\left\langle F_{p} / N_{p}\right\rangle$ and the proof of the first equality is complete.

For the second claim in the statement of the proposition, the containment one way is clear. So let $G \in\langle\mathscr{G} \mathcal{V}\rangle \cap \mathcal{G}$. Although we generally restrict $X$ to have cardinality $\boldsymbol{\aleph}_{0}$, Lemma 3.2 and the above argument remain valid for $|X| \geqslant \boldsymbol{\aleph}_{0}$. So suppose that we take $|X|=\max \left\{|G|, \boldsymbol{\kappa}_{0}\right\}$. A free object in $\mathscr{G T}$ is $S=$ TR $\left(I, F_{p} / N_{p}, I ; P / N_{p}\right)$. Since $\mathcal{V} \in \mathcal{L}(\mathscr{D})$, it follows from Lemma 3.2 that $N_{p}$ is a fully invariant subgroup of $F_{p}$ and therefore that $F_{n} / N_{p}$ is a relatively free object in $\left\langle F_{p} / N_{p}\right\rangle$ on a set of cardinality $|X|$. Hence, by the first part of the proposition, there exists a normal subgroup $K$ of $F_{p}$ such that $\left(F_{p} / N_{p}\right) /\left(K / N_{p}\right)$ is isomorphic to $G$. For $\rho=\rho_{K / N_{p}}$ we then have

$$
S / \rho \cong \Re\left(I,\left(F_{p} / N_{p}\right) /\left(K / N_{p}\right), I ;\left(P / N_{p}\right) /\left(K / N_{p}\right)\right)
$$

with structure group isomorphic with $G$. Hence $G$ itself will be a subgroup of some element of $\mathscr{T} \widetilde{V}$ and the second claim is established.

We are now ready for the desired homomorphism.

THEOREM 3.8. The mapping

$$
\gamma: \mathcal{V} \rightarrow\langle\mathscr{V}\rangle \cap \mathbb{Q S} \quad(\mathcal{V} \in \mathcal{L}(\mathscr{D}))
$$

is a homomorphism of $\mathcal{L}(\mathscr{Q})$ onto $\mathcal{L}(\mathbb{Q})$.

Proof. First consider the interval $[R \mathscr{G}, \mathcal{C}]$.

(i) The mapping $\mathscr{V} \rightarrow \rho(\mathcal{V})$ is an anti-isomorphism of [ $R \mathscr{R}, \mathscr{D}]$ onto the interval $[\rho(\mathscr{D}), \rho(\Re \Re)]$ of fully invariant congruences on $F$, by Proposition 2.8 .

(ii) The mapping $\rho(\mathcal{V})=\rho_{N} \rightarrow N$ is an isomorphism of $[\rho(\mathscr{D}), \rho(\Re \mathscr{B})]$ onto the interval $\left[\hat{F}_{p}^{\prime}, F_{Z}\right]$, by Propositions 2.16 and 2.18 .

(iii) By Lemma 2.11 (iii), $\pi_{p}$ induces a homomorphism of the interval $\left[\hat{F}_{p}^{\prime}, F_{Z}\right]$ into $\Re_{p}$ and clearly must map this interval onto the interval $\left[F_{p}^{\prime}, F_{p}\right]$ of $\Re_{p}$. 
(iv) By Lemma 3.2, the interval $\left[F_{p}^{\prime}, F_{p}\right]$ of $\mathscr{K}_{p}$ is precisely the interval $\left[F_{p}^{\prime}, F_{p}\right]$ in the lattice of fully invariant subgroups of $F_{p}$. Since $F_{p}$ is a free group on a countable number of generators, there is an anti-isomorphism $N_{p} \rightarrow\left\langle F_{p} / N_{p}\right\rangle$ of the interval $\left[F_{p}^{\prime}, F_{p}\right]$ onto the lattice of varieties of abelian groups.

Combining the mappings (i)-(iv), we find that the mapping

$$
\Upsilon \rightarrow\left\langle F_{p} / N_{p}\right\rangle,
$$

where $\rho(\mathscr{V})=\rho_{N}$, is a homomorphism of $[\mathscr{R} \mathscr{B}, \mathscr{D}]$ into $\mathcal{L}(\mathscr{Q S})$. For $\mathscr{Q} \in \mathcal{L}(\mathbb{Q})$, let $N=\mathscr{U}\left(F_{Z}\right)$ (see the beginning of $\S 2$ ). Then $N \in \mathcal{N}$ and, if $\mathscr{V}=\left\langle F / \rho_{N}\right\rangle$ it is straightforward to see that $\mathscr{Q}=\left\langle F_{p} / N_{p}\right\rangle$ and the mapping (4) is an epimorphism.

By Proposition 3.7, we now have an epimorphism $\gamma: \mathcal{T} \rightarrow\langle\mathscr{G}\rangle \cap \mathbb{Q} \mathcal{G}$ of $[\mathscr{R} \mathscr{\mathcal { B }}, \mathscr{Q}]$ onto $\mathscr{L}(\mathscr{Q S})$. If we extend $\gamma$ to $\mathscr{L}(\mathscr{D})$ by defining $\mathscr{V} \gamma=\mathcal{T}$, the trivial variety, for all $\mathcal{V} \subseteq \mathcal{R}$, , it is easy to see that this extension is a homomorphism and is the mapping in the statement of the theorem.

A further homomorphism of $\mathcal{L}(\mathscr{Q})$ follows.

THEOREM 3.9. The mapping

$$
\eta: \mathcal{T} \rightarrow(\langle\mathscr{T}\rangle \cap \mathcal{Q P}, \mathcal{T} \cap \mathcal{G}) \quad(\mathcal{T} \in \mathcal{L}(\mathscr{Q}))
$$

is a homomorphism of $\mathcal{L}(\mathscr{Q})$ onto the subdirect product

$$
\mathfrak{R}=\{(\mathcal{Q}, \mathfrak{T}) \in \mathcal{L}(\mathfrak{Q S}) \times \mathfrak{L}(\mathcal{G}) \mid \mathcal{Q} \subseteq \mathfrak{T}\} .
$$

The restriction of $\eta$ to the interval $[R \mathscr{R}, \mathcal{C}]$ is an isomorphism onto the lattice $\mathscr{R}$.

Proof. For $\mathscr{V} \in \mathcal{L}(\mathscr{D})$, with $\rho(\mathcal{V})=\rho_{N}$, it follows from Proposition 2.18 that $F_{p}^{\prime} \subseteq N_{p}$ and from Proposition 3.7 that every group in $\langle G \mathscr{V}\rangle$ is abelian. Consequently, $\langle\mathscr{G} \mathcal{V}\rangle \cap \mathbb{Q P}=\langle\mathscr{G} \mathcal{V}\rangle \cap \mathcal{G}$. It now follows from Theorem 3.8 and Theorem 2.12 , that $\eta$ is a homomorphism while the range of $\eta$ is clearly contained in $R$.

Let $(\mathcal{Q}, \mathcal{V}) \in \Re$ and let $L$ (respectively, $M$ ) be the fully invariant subgroup of $F_{Z}$ corresponding to $\mathscr{Q}$ (respectively, $\mathscr{V}$ ). Then $M \subseteq L$. Let $N=M\left[F_{Z}, F_{p}\right] L_{p}$. Now $M$ and $\left[F_{Z}, F_{p}\right]$ are normal subgroups of $F_{Z}$ while, for any $a \in F_{Z}, b \in L_{p}$, $a^{-1} b a=\left(a^{-1} b a b^{-1}\right) b \in\left[F_{Z}, F_{p}\right] L_{p}$. Hence $N$ is a normal subgroup of $F_{Z}$. Each of the subgroups $M,\left[F_{Z}, F_{p}\right]^{\wedge}$ and $L_{p}$ is clearly invariant under elements of $\mathscr{E}\left(F_{Z}\right)$ and therefore so is $N$. Hence $N \in \mathcal{X}$. Let $\mathscr{W}$ be the variety such that $\rho(\mathcal{Q})=\rho_{N}$. Since $\left[F_{Z}, F_{p}\right] \subseteq N$, it follows from Proposition 2.14 that $\mathscr{Q} \in \mathcal{L}(\mathcal{C})$. Now, since $F_{p} \pi_{q}=$ $\{1\}, N_{q}=N \pi_{q}=M \pi_{q}=M_{q}$ while $N_{p}=N \pi_{p}=M_{p}\left[F_{p}, F_{p}\right] L_{p}=L_{p}$ since $M_{p} \subseteq L_{p}$ (because $M \subseteq L$ ) and $F_{p}^{\prime} \subseteq L_{p}$ (because $\mathscr{Q} \in \mathcal{L}(\mathscr{Q S})$ ). By Proposition 3.7 and Theorem 2.12, $\mathscr{V} \eta(\mathscr{U}, \mathscr{V})$. Thus $\eta$ maps $[\mathcal{R} \mathscr{B}, \mathcal{C}$ ] onto $R$.

Finally, it remains to show that $\eta$ is one-to-one on $[\Re \mathscr{B}, \mathcal{C}$. Let $\mathcal{Q}, \mathcal{V} \in[\Re \mathscr{R}, \mathcal{C}]$ and $\mathscr{U} \eta=\mathscr{V} \eta$ where $\rho(\mathcal{U})=\rho_{M}, \rho(\mathcal{V})=\rho_{N}$. By Proposition 3.7 and Theorem 2.12, we have $M_{p}=N_{p}$ and $M_{q}=N_{q}$. But Proposition 2.14 implies that $\left[F_{Z}, F_{p}\right]$ $\subseteq M \cap N$ and so, from Lemma 3.3, we must have $M=N$.

REMARK 3.10. A precise description of the variety $\delta \in[\Re \mathscr{G}, \varrho]$ for which $\delta \eta=(\mathscr{Q}, \mathcal{V})$ for a prescribed $(\mathcal{Q}, \mathcal{V}) \in \mathscr{R}$ will be given in Theorem 4.3.

We are finally ready for our main result for the hardest part of the argument has been completed, and we have only to put the pieces together. 
THEOREM 3.11. The mapping

$$
\zeta: S \rightarrow(S \cap G \mathscr{G},\langle\mathscr{S} S\rangle \cap \mathbb{Q} \mathcal{G}, S \cap \mathcal{G}) \quad(S \in \mathcal{L}(\mathscr{D}))
$$

is a homomorphism of $\mathcal{L}(\mathcal{D})$ onto the subdirect product

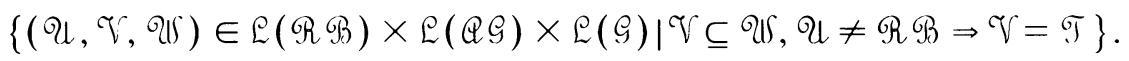

Its restriction to $\mathcal{L}(\mathcal{C})$ is an isomorphism of $\mathcal{L}(\mathcal{C})$ onto this subdirect product.

Proof. Denote by $\mathscr{P}$ the last set in the theorem. If we write $\delta \zeta=(\mathcal{Q}, \mathcal{V}, \mathcal{Q} S)$, then trivially $\mathcal{V} \subseteq \mathcal{W}$, and if $\mathscr{U} \neq \mathscr{R} \Re$, then $S$ is either a left group variety or a right group variety or a group variety, and in each of these cases $\mathscr{V}=\langle G S\rangle \cap Q \mathcal{Q}=\mathcal{T}$. Hence $\zeta$ maps $\mathcal{L}(\mathscr{D})$ into $\mathscr{P}$.

The mapping $\zeta$ is a homomorphism in view of Lemma 3.1 and Theorem 3.9. Let $\Re, \delta \in R(C)$ and assume that $R \zeta=\delta \zeta$. If $\Re \cap \Re \Re=\delta \cap \Re \Re=\Re \Re$, then

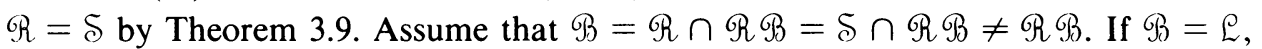
the variety of left zero semigroups, then $R=\varrho \vee R^{\prime}, \delta=\varrho \vee S^{\prime}$ for group varieties $\Omega^{\prime}$ and $S^{\prime}$. But then

$$
\Re^{\prime}=\left(\mathcal{L} \vee \Re^{\prime}\right) \cap \mathcal{G}=\left(\mathfrak{L} \vee S^{\prime}\right) \cap \mathcal{G}=S^{\prime}
$$

so that $R=\delta$; the case of right group varieties is symmetric, and of group varieties is trivial. Therefore $\zeta$ is one-to-one on $\mathcal{L}(\mathcal{C})$.

Let $(\mathscr{Q}, \mathcal{T}, \mathscr{W}) \in \mathscr{P}$. Assume first that $\mathscr{Q}=\Re \Re$. By Theorem 3.9, there exists a variety $\delta \in[R \mathscr{R}, \mathcal{C}]$ such that $\delta \eta=(\mathcal{V}, \mathscr{W})$ which evidently implies that $\delta \zeta=$ $(\mathscr{Q}, \mathcal{T}, \mathcal{Q}$ ). Suppose next that $\mathscr{Q} \neq \Re \mathscr{R}$. Then $\mathcal{V}=\mathcal{T}$, so letting $\delta=\mathscr{Q} \vee \mathscr{W}$, we easily verify that $\delta \zeta=(\mathscr{Q}, \mathcal{V}, \mathscr{Q})$. Consequently, $\zeta$ maps $\mathcal{L}(\mathcal{C})$ onto $\mathscr{P}$.

4. Certain properties of the embedding of $\mathcal{L}(\mathcal{C})$. Let $\delta \in \mathcal{L}(\mathcal{C})$ and $\delta \zeta=$ $(\mathcal{Q}, \mathcal{T}, \mathcal{Q})$. If $\mathscr{Q}, \mathcal{T}$ and $\mathscr{W}$ are given by systems of identities, we construct here a system of identities for $\mathcal{S}$. Next we characterize the completely simple semigroups belonging to $\delta$ in two different ways in terms of the varieties $\mathcal{Q}, \mathcal{V}$ and $\mathcal{Q}$. We then describe how $\delta$ can be built from the varieties $\mathcal{U}, \mathcal{V}$, and $\mathcal{W}$ in terms of transformations of these varieties. We also construct a retraction of $\mathcal{L}(\mathcal{D})$ onto $\mathcal{L}(\mathcal{C})$, and characterize the congruence on $\mathcal{L}(\mathscr{D})$ induced by $\zeta$.

Notation 4.1. Let $\mathscr{U}=[u=v]$ be a variety of rectangular bands. Replace each variable $x$ occurring in $u$ or $v$ by $x^{0}=x x^{-1}$ and denote the resulting identity by $u^{*}=v^{*}$.

Let $\mathcal{V}$ be a variety of abelian groups. It is well known that $\mathcal{T}=\mathscr{Q S}$ or $\mathcal{T}=\left[x^{k}=1\right]$ for some natural number $k$. In any case, $\mathcal{T}=\left[w=z, x=y x y^{-1}\right]$ in the variety of completely simple semigroups, where $w=z$ is either $x=x$ or $x^{k}=x^{0}$. For the case $x=x$, let $w_{n}=z_{n}$ stand for the identity $x=x$ for all $n \geqslant 1$. For $x^{k}=x^{0}$, for every $n \geqslant 1$, let $w_{n}=z_{n}$ stand for the identity $\left(x_{1}^{0} x_{2}^{0} \cdots x_{n}^{0}\right)^{k}=\left(x_{1}^{0} x_{2}^{0} \cdots x_{n}^{0}\right)^{0}$. $\left.\cdots x_{n}^{0}\right)^{0}$.

Let $\mathcal{Q}=\left[s_{\alpha}=t_{\alpha}\right]_{\alpha \in A}$ be a variety of groups. Fix a variable $r$, and replace each variable $x$ occurring in $s_{\alpha}$ or $t_{\alpha}$ by the variable $\bar{x}=r^{0} x r^{0}$. Denote the identity $s_{\alpha}\left(\bar{x}_{i}\right)=t_{\alpha}\left(\bar{x}_{i}\right)$ by $\bar{s}_{\alpha}=\bar{t}_{\alpha}$.

We are now ready for the first part of our programme. 
THEOREM 4.2. Let

$$
\begin{gathered}
\mathscr{Q}=[u=v, x=x y x], \quad q=\left[w=z, x=y x y^{-1}\right], \\
\mathscr{Q} \int=\left[s_{\alpha}=t_{\alpha}, x x^{-1}=y y^{-1}\right]_{\alpha \in A} .
\end{gathered}
$$

Then

$$
\checkmark=\left\{S \in \mathcal{Q} \mid S / \mathcal{H} \in \mathcal{Q} \text {, subgroups of }\left[E_{s}\right] \text { lie in } \mathcal{T}, \text { subgroups of } S \text { lie in } \mathscr{Q} S\right\} .
$$

coincides with the variety

$$
\left[a x a^{0} y^{0} a=a y^{0} a^{0} x a, u^{*}=v^{*}, w_{n}=z_{n}, n=1,2, \ldots, \bar{s}_{\alpha}=\bar{t}_{\alpha}, \alpha \in A\right] .
$$

Proof. First let $S \in \Omega$. Then $S / \mathcal{H} \in \mathcal{Q l}$ and hence $S / \mathcal{H}$ satisfies the identity $u=v$. Then $u=v$ can be taken to be one of the identities: $x=y, x y=x, x y=y$, $x=x$. In the first case, $S$ is a group and thus satisfies the identity $x x^{-1}=y y^{-1}$. In the second case, $S$ is a left group and thus satisfies the identity $x y y^{-1}=x$, which is equivalent to the identity $x x^{-1} y y^{-1}=x x^{-1}$. The case $x y=y$ is similar, and the case $x=x$ amounts to $x x^{-1}=x x^{-1}$. Consequently, $S$ satisfies the identity $u^{*}=v^{*}$.

If $w=z$ is equivalent to $x=x, S$ trivially satisfies $w_{n}=z_{n}$ for all $n$. Let $w=z$ be the identity $x^{k}-1$, and consider the identity $z^{k}=z^{0}$ where $z=x_{1}^{0} x_{2}^{0} \cdots x_{n}^{0}$. For any substitution of the variables $x_{i}$ by elements of $S$, the resulting element $z$ lies in [ $E_{S}$ ], and hence $z^{k}$ is the identity of the maximal subgroup of $S$ containing $z$. Thus $S$ satisfies the identity $z^{k}=z^{0}$, that is to say, $S$ satisfies the identity $w_{n}=z_{n}$.

Now consider an identity $s_{\alpha}=t_{\alpha}$ figuring in the definition of $\mathscr{Q} \int$. Substituting the variables $x$ in $s_{\alpha}$ or $t_{\alpha}$ by $\bar{x}=r^{0} x r^{0}$, we obtain the identity $\bar{s}_{\alpha}=\bar{t}_{\alpha}$ over variables contained in some maximal subgroup. Since $s_{\alpha}=t_{\alpha}$ is a law in Q Of and $\bar{s}_{\alpha}=\bar{t}_{\alpha}$ coincides with $s_{\alpha}=t_{\alpha}$ when all variables are contained in a subgroup, we conclude that $S$ satisfies the identity $\bar{s}_{\alpha}=\bar{t}_{\alpha}$.

Finally, since $S \in \mathcal{C}$, it must satisfy the identity $a x a^{0} y^{0} a=a y^{0} a^{0} x a$, by Proposition 2.14 .

Conversely, let $S$ be contained in the last set in the statement of the theorem. Since $S$ satisfies $u^{*}=v^{*}$, so does $S / \mathcal{H}$, which is a band, and thus $S / \mathcal{H}$ satisfies $u=v$. Consequently, $\delta / \mathcal{H} \in \mathcal{Q}$. Consider the identity $x^{k}=1$ for $w=z$. Every element of $\left[E_{S}\right]$ is of the form $x_{1}^{0} x_{2}^{0} \cdots x_{n}^{0}$. Since $S$ satisfies the identity $w_{n}=z_{n}$, we have $\left(x_{1}^{0} x_{2}^{0} \cdots x_{n}^{0}\right)^{k}=\left(x_{1}^{0} x_{2}^{0} \cdots x_{n}\right)^{0}$. This being true for all natural numbers $n$, we deduce that the subgroups of $\left[E_{S}\right.$ ] satisfy the identity $x^{k}=1$. Finally, $S$ satisfies the identities $\bar{s}_{\alpha}=\bar{t}_{\alpha}$, which in a subgroup of $S$ amount to the identities $s_{\alpha}=t_{\alpha}$. Therefore, $S \in \mathcal{S}$, as required.

As the second part of our programme, we have

THEOREM 4.3. If $\delta \in \mathcal{L}(\mathcal{C})$ and $\delta \zeta=(\mathcal{Q}, \mathcal{T}, \mathcal{Q} S)$, then

$$
\begin{aligned}
\mathcal{S} & =\left\{S \in \mathcal{C} \mid S / \mathcal{H} \in \mathcal{Q}, \text { subgroups of }\left[E_{S}\right] \text { lie in } \mathscr{V}, \text { subgroups of } S \text { lie in } \mathscr{W}\right\} \\
& =\left\{S \in \mathcal{C} \mid\langle S\rangle \cap \mathfrak{R} \mathscr{B} \subseteq \mathcal{Q},\left\langle\left[E_{S}\right]\right\rangle \cap \mathbb{Q S} \subseteq \mathcal{V},\langle S\rangle \cap \mathcal{G} \subseteq \mathscr{W}\right\} .
\end{aligned}
$$

Proof. Denote the second and third classes in the statement of the theorem by $\mathscr{P}$ and 2 , respectively. By Theorem $4.2, \mathscr{P}$ is a variety of central completely simple 
semigroups. In the light of Theorem 3.11 , to show that $\delta=\mathscr{P}$, it suffices to verify that

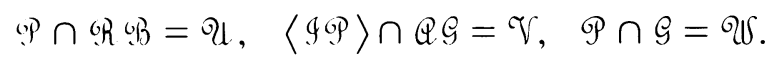

If $S \in \mathscr{P} \cap \mathscr{Q} \cap$, , then $S / \mathcal{H} \in \mathcal{Q}$ and $\mathcal{H}$ is the equality relation, so $S \in \mathcal{U}$. Conversely, if $S \in \mathcal{Q l}$, then obviously $S \in \mathscr{P} \cap \Re \Re$.

Let $S \in\langle\mathscr{P}\rangle \cap Q \mathcal{Q}$. By Lemma 2.19, there exist $S_{\alpha} \in \mathscr{9} \mathscr{P}$, a subdirect product $T \subseteq \Pi_{\alpha \in A} S_{\alpha}$, and an epimorphism $\varphi: T \rightarrow S$. For any $t \in T$, we have $t^{0} \varphi=1$, the identity of $S$. For any $s \in S$, there exists $p \in T$ such that $s=p \varphi$ and thus

$$
s=p \varphi=\left(t^{0} \varphi\right)(p \varphi)\left(t^{0} \varphi\right)=\left(t^{0} p t^{0}\right) \varphi .
$$

It follows that the mapping $\psi=\left.\varphi\right|_{H_{t}}$ is a homomorphism of the $\mathcal{G}$-class $H_{t}$ of $T$ onto $S$. Now $H_{t}$ is a subdirect product of its projections $K_{\alpha}$ in $S_{\alpha}$. Noting that $K_{\alpha} \in \mathcal{T}$, we conclude that $S \in \mathcal{T}$. Consequently, $\langle\mathscr{G} \mathscr{P}\rangle \cap \mathbb{Q} \mathscr{\mathcal { S }} \subseteq \mathcal{V}$.

In order to show the opposite inclusion, we may assume that $\mathscr{V} \neq \mathcal{T}$ so that o $R \subseteq S$. Let $K$ be the fully invariant subgroup of $F_{p}$ corresponding to $\mathcal{T}$. Then $F_{p} / K \in \mathscr{V} \subseteq \mathcal{O l l}$ and hence $\mathscr{N}\left(I, F_{p} / K, I ; P / K\right) \in \mathscr{P}$ which, being idempotent

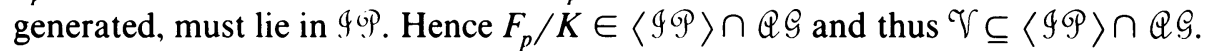

Trivially, $S \in\left(P \cap \mathcal{P}\right.$ if and only if $S \in \mathcal{Q} \int$.

We have verified (5) and thus have proved that $\mathcal{S}=\mathscr{P}$.

Next let $S \in \Re$. Let $K \in\langle S\rangle \cap \leftrightarrow R$. I . In view of Lemma 2.19, there is a subdirect product $T$ of copies $S_{\alpha}$ of $S$ and an epimorphism $\varphi: T \rightarrow K$. Let $T^{\prime}=\left\{\left(H_{s_{\alpha}}\right) \mid\left(s_{\alpha}\right) \in\right.$ $T\}$ and map $T^{\prime}$ to $K$ by $\psi:\left(H_{s_{\alpha}}\right) \rightarrow\left(s_{\alpha}\right) \varphi$. It follows at once that $\psi$ is single-valued, and thus a homomorphism of $T^{\prime}$ onto $K$. Since $T^{\prime}$ is a subdirect product of copies $S_{\alpha} / \mathcal{H}$ of $S / \mathcal{H}$, we deduce that $K \in \mathcal{Q}$.

Now let $K \in\left\langle\left[E_{S}\right]\right\rangle \cap \mathbb{Q} \mathcal{G}$. Similarly as above, there is a subdirect product $T$ of copies $T_{\alpha}$ of $\left[E_{S}\right]$ and an epimorphism $\varphi: T \rightarrow K$. As in the first part of the proof, $K$ is a homomorphic image of a subgroup of $T$, which in its turn is a subdirect product of its projections $P_{\alpha}$ in $T_{\alpha}$. Since all $P_{\alpha}$ are in $\mathcal{V}$, we conclude that $K \in \mathcal{V}$.

Finally, if $K \in\langle S\rangle \cap \mathcal{G}$, a similar argument can be used to prove that $K \in \mathcal{W}$.

We have proved that $S \in \mathcal{L}$ so that $P \subseteq \mathcal{2}$.

Now let $S \in \mathcal{2}$. Then $S / \mathcal{H} \in\langle S\rangle \cap \Re \Re \subseteq \mathcal{Q}$. If $G$ is a subgroup of [ $E_{S}$ ], then $G \in\left\langle\left[E_{S}\right]\right\rangle \cap \mathbb{Q} \mathcal{G} \subseteq \mathcal{V}$, and if $G$ is a subgroup of $S$, then $G \in\langle S\rangle \cap \mathcal{G} \subseteq \mathcal{W}$. Consequently, $S \in \mathscr{P}$ which shows that $\mathcal{Q} \subseteq \mathcal{P}$.

RemarK 4.4. Theorem 4.3 is particularly valuable when deriving the triple representation of Theorem 3.11 for particular varieties. We may readily deduce the following triple representations for certain standard varieties:

$$
\begin{aligned}
& \mathcal{G}=(\mathcal{T}, \mathcal{T}, \mathcal{G}), \quad \Re \mathscr{B}=(\Re \mathscr{B}, \mathcal{T}, \mathcal{T}), \\
& \Re \mathcal{G}=(\Re \mathscr{B}, \mathcal{T}, \mathcal{G}), \quad \Re \mathscr{S}=(\Re \mathscr{B}, \mathcal{T}, \mathscr{Q S}) \text {, } \\
& \mathcal{C}=(\Re \mathscr{B}, \mathbb{Q S}, \mathcal{G}), \quad \mathbb{Q}=(\Re \mathscr{B}, \mathbb{Q} \mathcal{G}, \mathbb{Q} \mathcal{G}) \text {. }
\end{aligned}
$$

Moving to the third part of our programme, we require the following notation from [6].

Notation 4.5. For $\mathscr{Q} \in \mathcal{L}(\mathcal{G})$, we will write $\overline{\mathscr{Q}}=\{S \in \mathcal{C S} \mid$ the subgroups of $S$ lie in $\mathscr{Q}$ \}. It is easily seen that $\overline{\mathscr{Q}}$ is a variety. 
COROLlary 4.6. Let $S \in \mathcal{L}(\mathcal{C})$ and $\delta \zeta=(\mathcal{Q}, \mathfrak{T}$, $\mathscr{W})$. Then

$$
\begin{array}{ll}
\varsigma=\mathscr{U} \vee \mathscr{Q} S & \text { if } \mathscr{A} \neq \Re \mathscr{B}, \\
\varsigma=\overline{\mathcal{V}} \vee \mathcal{U} S & \text { if } \mathscr{U}=\mathscr{R} \mathscr{B} .
\end{array}
$$

Proof. If $\mathscr{Q} \neq \mathscr{R} \mathscr{B}$, then $\mathcal{V}=\mathcal{T}$ and Theorem 4.3 yields

$$
\varsigma=\{S \in \Re \mathcal{G} \mid S / \mathcal{H} \in \mathcal{Q} \text {, subgroups of } S \text { lie in } \mathscr{W}\}=\mathscr{Q} \vee \mathcal{Q} \text {. }
$$

Assume next that $\mathscr{Q}=\mathscr{R} \mathscr{B}$. First note that by Theorem 4.3,

$$
(\mathscr{R} \mathscr{B}, \mathscr{V}, \mathscr{V}) \zeta^{-1}=\{S \in \mathcal{Q} \mid \text { subgroups of } S \text { lie in } \mathscr{V}\}=\overline{\mathcal{V}}
$$

Further,

$$
(\mathscr{Q}, \mathscr{V}, \mathscr{W})=(\mathscr{R}, \mathcal{V}, \mathscr{W})=(\Re \mathscr{R}, \mathscr{V}, \mathscr{V}) \vee(\mathcal{T}, \mathcal{T}, \mathscr{Q})
$$

and using Theorem 3.11, we may apply $\zeta^{-1}$ to both sides of this equation. Taking into account (6), we conclude that $\delta=\overline{\mathcal{T}} \vee$ $\int$.

REMARK 4.7. We provide here alternative forms for a variety $S$ in $\mathcal{L}(\mathcal{C})$ containing $\Re \Re$. Indeed

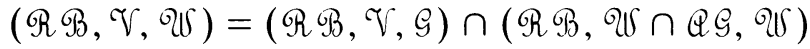

$$
\begin{aligned}
& =[(\Re \mathscr{T}, \mathcal{T}, \mathcal{G}) \vee(\Re \Re, \mathscr{T}, \mathcal{T})] \cap(\Re \mathscr{B}, \mathscr{W} \cap \mathbb{Q} \mathcal{G}, \mathscr{W})
\end{aligned}
$$

and thus applying $\zeta^{-1}$ we obtain: for $\mathcal{S} \in \mathcal{L}(\mathcal{C}), \mathcal{S} \supseteq \Re \Re$ and $\delta \zeta=(\Re \mathscr{B}, \mathcal{T}, \mathscr{Q}, \mathcal{S})$,

$$
\varsigma=(\Re \mathcal{G} \vee \overline{\mathcal{V}}) \cap(\overline{\mathcal{Q}} \cap \mathcal{C})=(\Re \mathcal{G} \vee \overline{\mathcal{V}}) \cap \overline{\mathcal{Q}}
$$

An interesting feature of the homomorphism $\zeta$ is as follows.

LEMMA 4.8. For any $\delta \in \mathcal{L}(\mathscr{D})$, we have $\delta \zeta=(\delta \cap \mathcal{C}) \zeta$.

Proof. It suffices to consider $\mathcal{S} \in \mathcal{L}(\mathscr{D}), \Re \mathscr{R} \subseteq \mathcal{S}$. Then

$$
\begin{aligned}
\delta \zeta & =(\Re \mathscr{T},\langle\mathscr{S}\rangle \cap \mathcal{Q P}, \delta \cap \mathcal{G}), \\
(\delta \cap \mathcal{C}) \zeta & =(\Re \mathscr{B},\langle\mathscr{G}(\delta \cap \mathcal{C})\rangle \cap \mathbb{Q},(\delta \cap \mathcal{C}) \cap \mathcal{G}),
\end{aligned}
$$

so that, in order to prove the desired equality, it suffices to show that $\langle 9 \mathcal{S}\rangle \cap \mathbb{Q S} \subseteq$ $\langle\mathscr{S}(\mathcal{S} \cap \mathcal{C})\rangle \cap \mathbb{Q S}$.

Let $G \in\langle 9 \mathcal{S}\rangle \cap \mathbb{Q} \mathcal{G}$. In the light of Lemma 2.19, there exists a subdirect product $T$ of semigroups $S_{\alpha}$ in $9 \mathcal{S}$ and an epimorphism $\varphi: T \rightarrow G$. We may take $S_{\alpha}=$ T⿱ $\left(I_{\alpha}, G_{\alpha}, \Lambda_{\alpha} ; P_{\alpha}\right)$ with $P_{\alpha}$ normalized. Then $G_{\alpha}$ is generated by the sandwich matrix entries, since $S_{\alpha}$ is idempotent generated. Since these entries commute, the group $G_{\alpha}$ is abelian. It follows that $S_{\alpha} \in \mathbb{Q} \subseteq \mathcal{C}$. Consequently, $S_{\alpha} \in \mathcal{S} \cap \mathcal{C}$, which completes the proof.

COROllary 4.9. The mapping

$$
\delta \rightarrow \delta \cap \mathcal{E} \quad(\delta \in \mathcal{L}(\mathscr{D}))
$$

is a retraction of $\mathcal{L}(\mathcal{D})$ onto $\mathcal{L}(\mathcal{C})$.

Proof. It suffices to observe that by Lemma 4.8, $\delta \rightarrow \delta \zeta=(\delta \cap \mathcal{C}) \zeta \rightarrow \delta \cap \mathcal{C}$ is the stated retraction.

We now turn to the congruence induced by $\zeta$ on $\mathcal{L}(\mathcal{D})$. To this end, we require the following relation $\gamma$ on $\mathcal{L}(\mathcal{C S})$ which was introduced in [7] where it was shown to be a congruence. 
Definition 4.10. The relation $\gamma$ is defined on $\mathcal{L}(\mathcal{S})$ as follows: $\mathscr{Q} \gamma \mathcal{V} \Leftrightarrow q \mathcal{q}=$ $9 \mathscr{Q}$.

Proposition 4.11. The mapping

$$
S \rightarrow(S \cap \Re \mathscr{R},\langle 9 S\rangle \cap Q \mathcal{G}) \quad(\delta \in \mathcal{L}(\mathscr{D}))
$$

is a homomorphism of $\mathcal{L}(\mathcal{D})$ onto the subdirect product

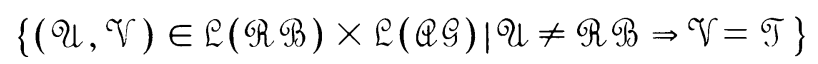

which induces $\gamma_{(\mathbb{R}(0))}$.

Proof. The first assertion is a consequence of Theorem 3.11. The second claim is equivalent to the following statement: for any $R, \delta \in \mathcal{L}(\mathscr{D})$,

$$
\mathscr{T}=\mathscr{G S} \Leftrightarrow \Re \cap \Re \mathscr{B}=S \cap \Re \mathscr{R}, \quad\langle\mathscr{R}\rangle \cap \mathbb{Q P}=\langle 9 \mathcal{S}\rangle \cap \mathbb{Q P} .
$$

First assume the validity of the left-hand side of (7). If $S \in \Re \cap \Re \mathscr{B}$, then $S \in \mathscr{R}=9 S$ so $S \in \mathcal{S} \cap \Re \Re$. Hence $\Re \cap \Re \Re \subseteq \delta \cap \Re \Re$ and the equality follows by symmetry.

Now suppose the validity of the right-hand side of (7) and let $S \in \mathscr{9}$. If $S \in \Re \mathscr{B}$, then $S \in \mathscr{R} \cap \Re \Re=\mathcal{S} \cap \Re \Re$ so $S \in \mathscr{S}$. Assume that $S \notin \Re \Re$. We may take $S=\mathfrak{R}(I, G, \Lambda ; P)$ where $P$ is normalized and thus $G$ is generated by the entries of $P$, so $G$ must be abelian. Hence

$$
G \in\langle G \Re\rangle \cap \mathbb{Q S}=\langle 9 S\rangle \cap \mathbb{Q P}
$$

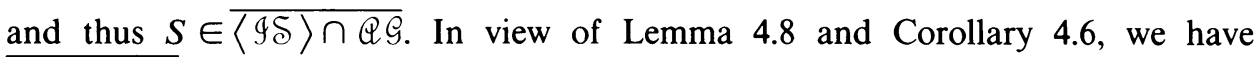
$\overline{\langle\mathscr{S}\rangle \cap \mathbb{Q S}} \subseteq \mathcal{S} \cap \mathcal{C} \subseteq \mathcal{S}$ so that $S \in \mathscr{S}$. Consequently, $\mathscr{R} \subseteq 9 \mathcal{S}$, and the equality follows by symmetry.

COROllary 4.12. The homomorphism $\zeta$ induces $\alpha \cap \gamma$ on $\mathcal{L}(\bigotimes)$.

Proof. It suffices to recall Theorem 3.11 and the definitions of $\alpha$ (in Theorem 2.12) and $\gamma$ (in Definition 4.10).

5. The structure of certain intervals of $\mathcal{L}(\mathcal{C})$. As an application of Theorem 3.11 and using the particular representations of Remark 4.4, we will consider here certain intervals which will amount to taking all triples satisfying some additional restrictions.

In the triple representation of varieties in $\mathcal{L}(\mathcal{C})$ figuring in Theorem 3.11 , fixing the first component to be $\Re \mathscr{B}$ and the second to be $\mathscr{Q S} \mathcal{S}$ and $\mathcal{T}$, respectively, we obtain the following result by allowing the third component to assume all permissible values.

Proposition 5.1. (i) $[\mathbb{Q}, \mathcal{C}] \cong[\mathfrak{Q} \mathcal{G}, \mathcal{G}]$.

(ii) $(\mathscr{R} \mathscr{B}, \mathcal{T}, \mathscr{W})\left(\gamma_{\mathfrak{L}(\mathcal{C})}\right) \cong[\mathcal{V}, \mathcal{G}]$.

Again fixing the first component to be $\Re \Re$, but fixing the third component to be $\mathcal{G}, \mathcal{Q S}$ and $\mathscr{W}$, respectively, while allowing the second component to assume all permissible values, we obtain the following additional isomorphisms. 
Proposition 5.2. (i) [ $\mathscr{R} \mathcal{G}, \mathcal{C}] \cong \mathscr{L}(\mathscr{Q} \mathcal{G})$.

(ii) $[R \mathscr{Q S}, \mathbb{Q}] \cong \mathscr{L}(\mathcal{Q S})$.

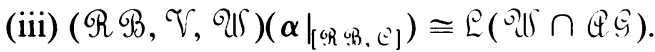

We now fix the first component to be $\mathscr{R} 93$ and restrict the third component to contain $Q \mathcal{G}$ (this removes the restrictions concerning the subdirect product). This leads to yet another isomorphism.

Proposition 5.3. [RQG, $\mathcal{Q}] \cong \mathscr{L}(\mathcal{Q}) \times[Q \mathcal{G}, \mathcal{G}]$.

Different types of isomorphisms for various intervals of the lattice $\mathcal{L}(\mathcal{C})$ can be devised. As an example of this, we prove the following result.

Proposition 5.4. (i) The mapping

$$
\kappa: \delta \rightarrow(\delta \cap \mathcal{Q}, \varsigma \cap \mathcal{G}) \quad(\delta \in[\mathcal{Q}, \mathcal{Q}])
$$

is an isomorphism of $[\mathcal{Q}, \mathcal{Q}]$ onto $[\mathcal{Q}, \mathbb{Q}] \times[\mathcal{Q}, \mathcal{Q}]$.

(ii) The mapping

$$
S \rightarrow(S \cap Q, S \cap \mathcal{R} \mathcal{G}) \quad(\delta \in[\mathscr{R Q G}, \mathcal{C}])
$$

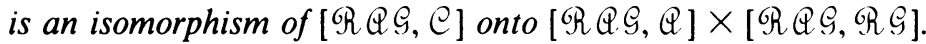

Proof. (i) Varieties in $[Q \mathcal{Q}, \mathcal{C}]$ correspond to the triples of the form ( $\left.91, \mathcal{Y}, Q_{1}\right) \in$

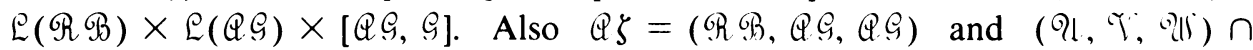
$(\mathscr{R} \mathscr{B}, \mathscr{Q S}, \mathscr{Q P})=(\mathscr{Q}, \mathcal{T}, \mathbb{Q} \mathcal{G})$. Hence the mapping $s \rightarrow S \cap \mathbb{Q}$ in the triple notation becomes

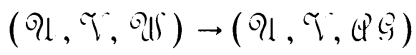

and thus it is obviously a homomorphism. By Theorem 2.12, the mapping $s \rightarrow s \cap S$ is a homomorphism, and hence so is $\kappa$. It follows from (8) that $\kappa$ is one-to-one. Since

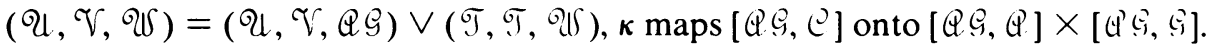

(ii) The argument here is similar to the above and is omitted.

\section{REFERENCES}

1. A. H. Clifford, The free completely regular semigroup on a set, J. Algebra 59 (1979), 434-451.

2. J. M. Howie, An introduction to semigroup theor, Academic Press, London, 1976

3. J. Leech, The structure of a band of groups, Mem. Amer. Math. Soc. 1 (1975), No. 157, pp. 67-95.

4. G. I. Maševickiī, On identities in varieties of completely simple semigroups over abelian groups, Contemporary Algebra, Leningrad, (1978), 81-89. (Russian)

5. M. Petrich, Introduction to semigroups, Merrill, Columbus, Ohio, 1973.

6. M. Petrich and N. R. Reilly, Varieties of groups and of completely simple semigroups, Bull. Austral. Math. Soc. 23 (1981), 339-359.

7. Near varieties of idempotent generated completely simple semigroups, Algebra Universalis (to appear).

8. V. V. Rasin, On the lattice of varieties of completely simple semigroups, Semigroup Forum 17 (1979), $113-122$.

9. Free completely simple semigroups, Mat. Zapiski Ural. Univ. 11 (1979), 140-151. (Russian)

Department of Mathematics, Simon Fraser University, Burnaby, British Columbia, Canada V5A 1 S6 